\title{
Domain Structure of Titanomagnetites and Its Variation with Temperature
}

\author{
H.C. SOFFEL \\ Institut für Allgemeine und Angewandte Geophysik, Universität München, \\ Theresienstrasse, München, Germany
}

(Received June 20, 1977)

In a Tertiary lava flow with optically apparently homogeneous titanomagnetites (Curie temperatures less than $100^{\circ} \mathrm{C}$ ) a range of Curie temperatures from less than $-40^{\circ} \mathrm{C}$ in the central to more than $70^{\circ} \mathrm{C}$ in the marginal zones of the crystals was found by observing the variation of the domain structure with temperature. The effect is explained by the different degree of maghemitization. TRM production in fields of 35 Oe revealed that the direction of the external field had little influence on the magnetic domain pattern.

\section{Introduction}

Thermoremanent magnetization (TRM) is produced in rocks during cooling in a magnetic field from high temperatures above the Curie temperature down to low temperature (about room temperature). The properties of TRM have been summarized recently by STACEY and BAnerJee (1974). Above the Curie temperature all ore grains are paramagnetic and have no domain configuration. By cooling below the Curie temperature saturation magnetization increases rapidly from practically zero to such high values that the energy balance of the crystal is strongly affected. In order to achieve a state of minimum energy, magnetic domains are developed. External fields, crystal anisotropy intensity of saturation magnetization, internal and external uniaxial stresses as well as the shape and irregularities of the external and internal surfaces of the crystal have an influence on the geometry, spatial distribution and size of the magnetic domains (see also STACEY and BANERJEE, 1974, p. 41). Each domain is magnetized more or less to saturation along an axis of easy magnetization. In the absence of an external field and of uniaxial stresses the domain configuration tends to be such that the net magnetic moment of the crystal is zero. External fields are believed to influence the development of magnetic domains in such a way that a remanence is produced. The sum of remanences of an aggregate of randomly oriented ore grains is in general assumed to be parallel to the direction of the external field. However the low intensity of TRM of true multidomain particles produced in a field of about $0.5 \mathrm{Oe}$ in comparison with the intensity of saturation remanence already indicates that small fields (say less than $1 \mathrm{Oe}$ ) seem to have little influence on the domain structure. It is generally believed that small external fields influence the domain structure mainly at or immediately below Curie temperature 
in such a way that the initially formed domains are magnetized in the easy magnetization direction which is closest to the direction of the external field.

The domain structure of natural titanomagnetites in basalts and its variation with temperature and direction of the external field during production of TRM was studied under the above mentioned aspects. The aim was to see how the domains develop in a small ore grain during cooling below Curie temperature.

\section{Rock Magnetic Properties of the Investigated Basalt}

For this special study a Tertiary basalt sample from the Southern Alps (flow No. $24 / 2$, SofFEL, 1975) was selected which contained titanomagnetites of apparently low oxidation state with bulk Curie temperatures of $100^{\circ} \mathrm{C}$ or less (Fig. 1). It was necessary to choose very low Curie temperature minerals because the sample could not be heated much above $100^{\circ} \mathrm{C}$ while under the microscope for technical reasons. Other rock magnetic properties of this lava flow have been published elsewhere (SoFFEL 1975). The ore grains appear to be optically homogeneous up to very large magnifications (1,300 times) before and after etching and show no signs of either deuteric oxidation or low temperature oxidation due to weathering. The bulk coercive force is about 70 Oe.

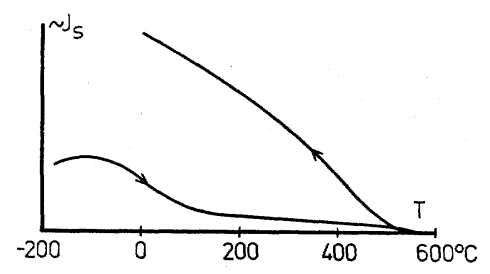

Fig. 1. Variation of saturation magnetization (measured in $5,000 \mathrm{Oe}$ ) with temperature between $-170^{\circ}$ and $+600^{\circ} \mathrm{C}$. Arrows indicate heating and cooling cycle. The increased intensities of the cooling cycle are due to exsolution of the titanomagnetites at high temperatures.

\section{Observation of Magnetic Domains}

It has been shown (SoFFEL, 1968, 1971) that the Bitter pattern technique is an adequate tool for the observation of magnetic domains of titanomagnetites in a rock matrix. Domain walls are observed on the polished surface of the crystals as dark lines. They represent the domain structure near the surface. In very small ore grains the observed domain structure on the surface can be regarded as representative for the interior of the crystal as well. Special care must be taken to get strainfree surfaces. This can be achieved by first mechanical and afterwards ionic polishing (Soffel and Petersen 1971). For the domain observation at various temperatures, an ester-based magnetite colloid ('Ferrofluid') was used instead of the magnetite colloid after Elmore (1938). Temperature could be varied between about $-50^{\circ} \mathrm{C}$ 

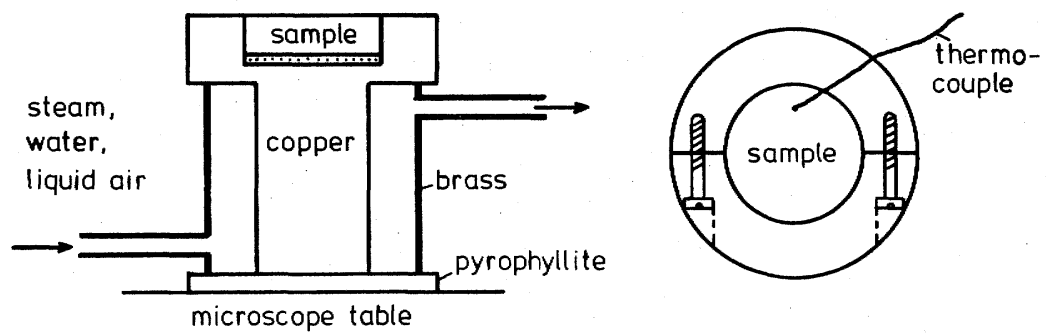

Fig. 2. Non-magnetic device for heating and cooling of polished sections while under the microscope. The cylindrical samples have a diameter of $2.5 \mathrm{~cm}$ and a height of about $1 \mathrm{~cm}$.

(freezing point of the immersion oil) and about $+100^{\circ} \mathrm{C}$ (highest temperature for the microscope objectives). Heating and cooling was done with the device shown in Fig. 2 by pumping liquid air, hot water or steam around the copper block. A thermocouple clamped to the sample surface was used to measure the temperature of the ore grains. External fields parallel to the surface of the polished section were produced with a small electromagnet. The field could be varied between $\pm 35 \mathrm{Oe}$ which is about 100 times the intensity of the horizontal component of the earth's field in the laboratory.

\section{Domains at Various Temperatures}

It could be concluded from the variation of saturation magnetization with temperature (Fig. 1) that only a certain fraction of the ore grains is ferrimagnetic at room temperature and should show domain structure (group I). The rest of the ore grains (group II) are supposed to have Curie temperatures below room temperature. They are paramagnetic and without domain configuration. Due to the optical homogeneity, all the ore grains should belong to either group I or II. However it was surprising to see that only the smallest ore grains with a diameter of less than about 6 microns were composed entirely of ferrimagnetic material and showed magnetic domains. The larger ore grains were only partly ferrimagnetic at room temperature, with large parts still paramagnetic, or they belonged to group II (no domain structure at all).

Figure 3a-3d shows a large ore grain with a diameter of about 60 microns at various temperatures. At room temperature (Fig. 3a) most of the crystal is above its Curie temperature with the exception of some small marginal zones which show a complicated domain structure. Details of this configuration shall not be discussed at the moment. At $70^{\circ} \mathrm{C}$ (Fig. 3b) most of these regions have become paramagnetic as well and lost their domain structure. After cooling to room temperature in a $35 \mathrm{Oe}$ field $(\rightarrow$ ), the domain structure (Fig. 3c) is not quite the same as before (see Fig. 3a) but the same zones are ferrimagnetic again. At $-40^{\circ} \mathrm{C}$, (Fig. 3d) most but not all of the crystal has become ferrimagnetic. A small grain, which showed no domains at $20^{\circ} \mathrm{C}$ and higher temperaturers, has become ferrimagnetic as well at $-40^{\circ} \mathrm{C}$ with a domain 


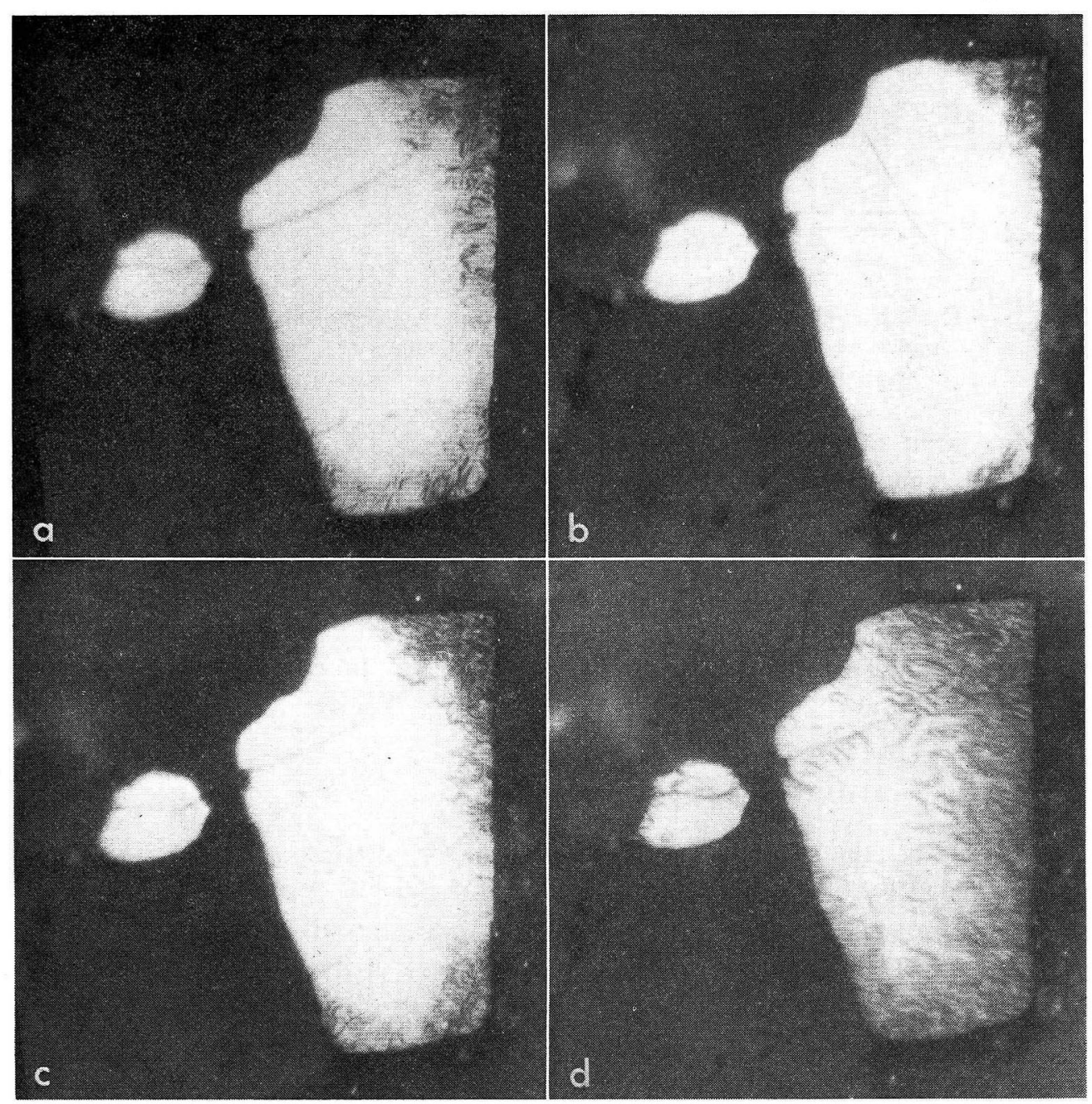

Fig. 3. Ore grain (diameter about 60 microns) at various temperatures. (a) room temperature, (b) $70^{\circ} \mathrm{C}$, (c) room temperature, (d) $-40^{\circ} \mathrm{C}$. For details, see text.

structure. The consequences of this range of Curie temperatures, from less than $-40^{\circ} \mathrm{C}$ to more than $+70^{\circ} \mathrm{C}$, in an optically apparently homogeneous crystal for TRM is discussed in the next section.

Figure $4 a-4 d$ shows a smaller titanomagnetite grain (length about 25 microns) which is almost entirely ferrimagnetic at room temperature (Fig. 4a). At $85^{\circ} \mathrm{C}$, the grain is paramagnetic and has lost its domain configuration (Fig. 4b). Cooling to room temperature in 35 Oe parallel to the surface $(\downarrow)$ reproduces substantially the original domain configuration (Fig. 4c). When the experiment is repeated with another direction of the external field of $35 \mathrm{Oe}(\rightarrow)$, the domain configuration is still not drastically altered (Fig. 4d). A schematic drawing of Fig. 4a-4d is shown in Fig. 5 . Dark lines are the observed domain wall traces. 


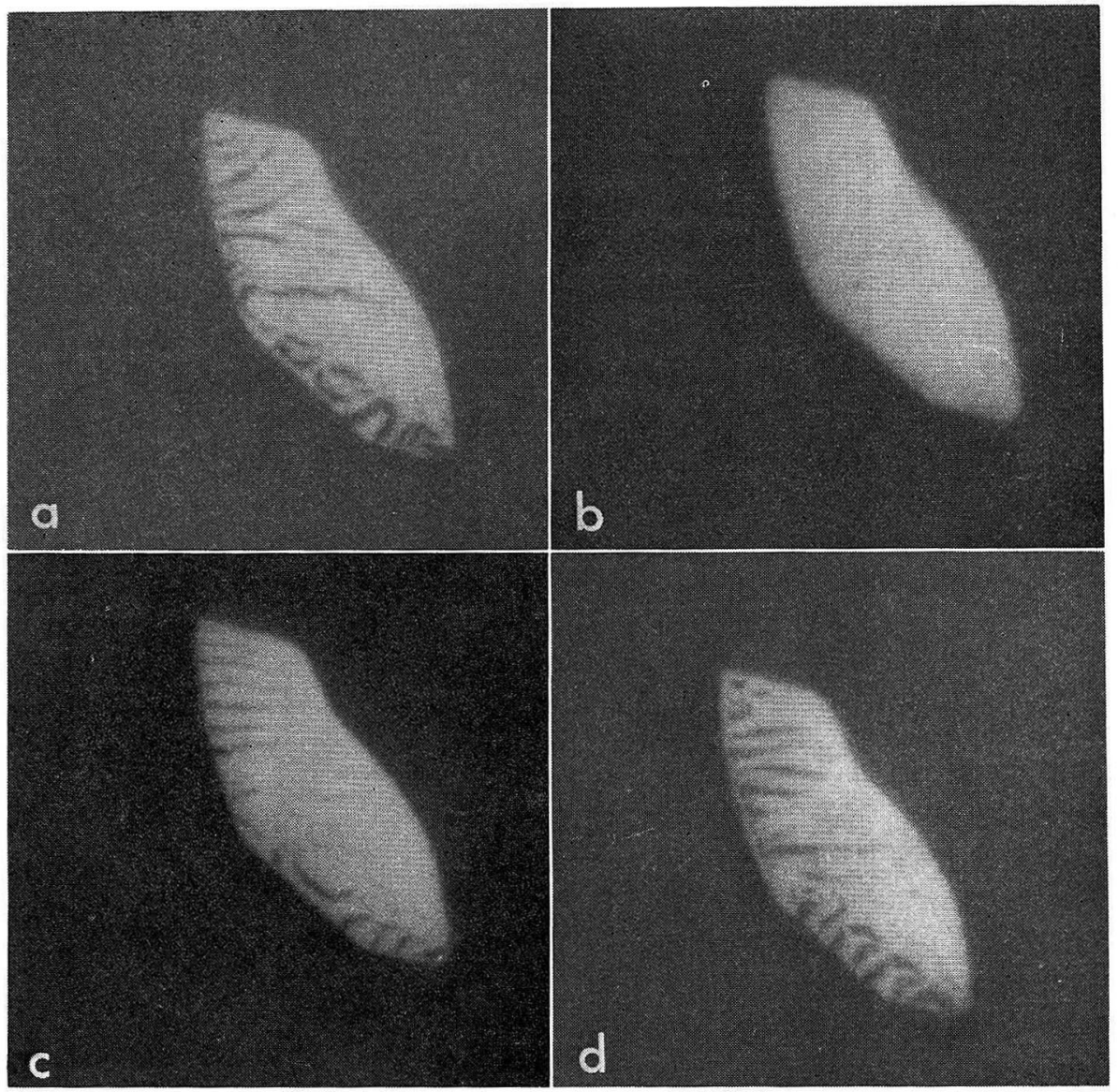

Fig. 4. Ore grain (length about 25 microns) at various temperatures. (a) room temperature, (b) $85^{\circ} \mathrm{C},(\mathrm{c})$ and (d) room temperature. For details, see text.

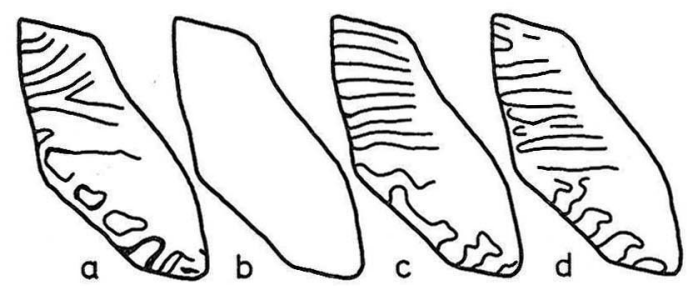

Fig. 5. Schematic drawing of the domain configurations shown in Fig. $4 a-d$.

Figure 6a-6f shows another small titanomagnetite grain (length about 10 microns). Figure 6 a represents the original domain configuration at room temperature, Figure $6 \mathrm{~b}$ shows the paramagnetic ore grain at $85^{\circ} \mathrm{C}$. After cooling in $35 \mathrm{Oe}$ parallel to the surface $(\rightarrow)$, almost the original configuration reappears (Fig. 6c). Reheating above Curie temperature and cooling in 35 Oe with a different horizontal 


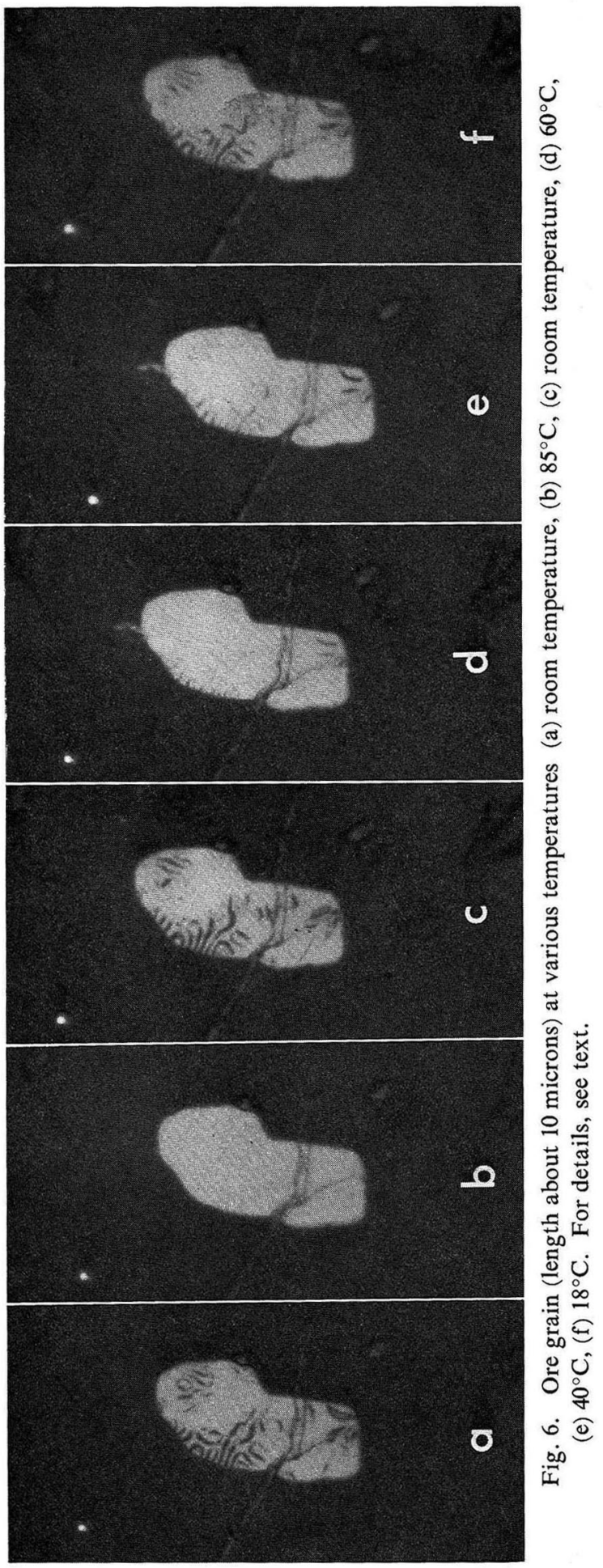


direction $(\downarrow)$ shows in Fig. $6 d$ the configuration at $60^{\circ} \mathrm{C}$, in Fig. 6e at $40^{\circ} \mathrm{C}$ and in Fig. $6 \mathrm{f}$ at $18^{\circ} \mathrm{C}$. The configurations of Fig. $6 \mathrm{a}, 6 \mathrm{c}$ and $6 \mathrm{f}$, though generated in different field directions, vary only in details in the upper right and central part of the crystal. It is difficult to estimate whether these differences are sufficient to produce differences in the net magnetic moment of the ore grain. On the other hand, the observed TRM in a rock must be composed of such individual effects in the ore grains. However, effects in a single ore grain may not necessarily be representative for a rock sample as a whole.

Figures 4-6 contain some surprising results. (i) Domain walls are not always parallel and straight. (ii) The domain magnetization is not always parallel to the long axis of the ore grain. (iii) The production of a TRM in a field one hundred times the earth's magnetic field does not influence the domain configuration very much.

Observations (i) and (ii) are common with titanomagnetites of low saturation magnetization. In pure magnetite, the domain walls tend to be straight and also the shape anisotropy influences the domain configuration in the expected way. The large intensity of saturation magnetization in combination with the moderate intensities of crystal anisotropy and magnetostrictive constants, $K$ and $\lambda$ respectively, prevent or reduce the occurrence of 'free magnetic charges' along curved domain walls due to local inhomogeneities and stress fields within the crystal or along the margins. This situation does not hold in low Curie temperature titanomagnetites with low values of saturation magnetization and large values of $K$ and $\lambda$ (SYoNo 1965). In titanomagnetites crystal anisotropy and magnetostrictive effects are more important and curved domain walls are often formed in order to reduce the energy of the ore grain at the expense of some additional magnetostatic energy along curved domain walls and along the margins of the crystal.

The most surprising observation to the author was the slight influence of rather strong external fields on the domain configuration during TRM production. Three possible explanations can be considered. (i) Although the domain configuration disappeared at a certain temperature on the visible part of a grain, it may still be present in other invisible parts of the ore grain. (ii) The Curie temperature was not completely attained but the saturation magnetization and the stray fields along the domain wall traces have become so small that they could no longer attract any particles of the magnetite colloid. (iii) The internal stress fields of the ore grains are so large that they have a stronger influence on the domain configuration than the external field applied $(35 \mathrm{Oe})$. In order to check the different possibilities, experiments with higher temperatures, larger external fields and various uniaxial stresses are planned.

\section{Discussion and Conclusions}

The very extended range of Curie temperature within an ore grain is believed to be due to maghemitization, with the highest degree of alteration along the margins and cracks. Such maghemitization does not affect the titanium-iron-ratio essentially 
and can therefore not be observed easily in microprobe studies. In X-ray analysis, it cannot be distinguished whether a certain diffraction line width is due to homogeneous grains with variable chemical composition or due to inhomogeneous ore grains. This may be the reason why the large range of Curie temperatures within the titanomagnetite grains was not detected earlier. The effect does not seem to be unique for the studied lava flow No. 24/2. Other basalts of the same region with very low Curie temperatures showed the same effect.

During the implacement of a volcanic rock the increased access of oxygen may not always lead to such a high oxidation state of titanomagnetites that exsolution into two different phases takes place. Lower oxidation states in the form of non stoichiometric titanomagnetites (titanomaghemites) may also occur, with the highest degree of maghemitization in the outer parts of the ore grains.

All theories about the origin of TRM assume a ferrimagnetic ore phase with a single Curie temperature or two separated phases with different Curie temperatures. These models seem to be realistic for rocks with magnetite or completely exsolved titanomagnetites, but not for rocks with primary non-stoichiometric titanomagnetites with decreasing Curie temperature from the margins towards the centre of the ore grain. It was shown in Fig. 3 how the domain configuration 'grows' steadily from the ferrimagnetic zones into the previously paramagnetic zones of the crystal during cooling. Their TRM is therefore eventually more influenced by the magnetic domains of the higher Curie temperature zones and their TRM information than by the weak external earth's magnetic field. This puts some question marks on the law of additivity of partial thermoremanent magnetizations on a microscopic scale in rocks with non-stoichiometric titanomagnetites. Figures 4-6 also show that the decay of intensity of remanence of basalts during thermal demagnetization may be only partly due to a spectrum of blocking temperature. A range of Curie temperatures, which can be as much as $100^{\circ} \mathrm{C}$, must also be considered.

The author is very much indebted to Dr. D. Dunlop for reading the paper at the fall 1976 meeting of AGU and for his helpful suggestions. Special thanks are due to the Munich rock magnetism group (Drs. Angenheister, Schult, Petersen, Pohl, Schmidbauer) for many useful discussions. The financial help of the Deutsche Forschungsgemeinschaft is gratefully acknowledged.

\section{REFERENCES}

Elmore, W.C., Ferromagnetic colloid for studying magnetic structures, Phys. Rev., 54, 309-310, 1938. SOFFEL, H., Die Bereichsstrukturen der Titanomagnetite in zwei tertiären Basalten und die Beziehung zu makroskopisch gemessenen magnetischen Eigenschaften dieser Gesteine, Habilitation Thesis, Fac. Nat. Sciences, University of Munich, Germany, 1-142, 1968.

SOFFEL, The single domain-multidomain transition in natural intermediate titanomagnetites, Z. Geophys., 37, 451-470, 1971.

SofFEL, H., Rock magnetism of the Monti Lessini and Monte Berici volcanites and age of volcanism deduced from the Heirtzler Polarity time Scale, J. Geophys., 41, 401-411, 1975.

Soffel, H. and N. Petersen, Ionic etching of titanomagnetite grains in basalts. Earth Planet. Sci. Lett., 11, 312-316, 1971.

StaCey, F.D. and S.K. BanerJee, The Physical Principles of Rock Magnetism, Elsevier Scientific Publishing Company, Amsterdam, London, New York, 1974.

SYONO, Y., Magnetocrystalline anisotropy and magnetostriction of $\mathrm{Fe}_{3} \mathrm{O}_{4}-\mathrm{Fe}_{2} \mathrm{TiO}_{4}$-series with special application to rock magnetism, Jpn. J. Geophys., 4, 71-143, 1965. 